\title{
Campylobacter jejuni
}

National Cancer Institute

\section{Source}

National Cancer Institute. Campylobacter jejuni. NCI Thesaurus. Code C86235.

A species of microaerophilic, Gram-negative, curved or spiral bacilli assigned to the phylum Proteobacteria. This species is motile with a flagellum at each pole, non-spore forming, oxidase positive, and hydrolyzes hippurate. C. jejuni is the most common pathogen in the United States causing bacterial diarrhea and gastroenteritis and is also linked to Guillain Barre syndrome. 